1 Hacettepe Journal of Mathematics and Statistics

$\bigcap$ Volume 47 (6) (2018), $1447-1452$

\title{
Lyapunov-type inequality for a Riemann-Liouville fractional differential boundary value problem
}

\author{
Maysaa Al-Qurashi* and Lakhdar Ragoub ${ }^{\dagger \ddagger}$
}

\begin{abstract}
The aim of this paper is to present a Lyapunov-type inequality for a Riemann-Liouville fractional differential equation of order $2<\alpha \leq 3$ subject to mixed boundary conditions.
\end{abstract}

Keywords: Lyapunov's inequality, Riemann-Liouville derivative, Caputo fractional derivative, mixed boundary conditions.

Mathematics Subject Classification (2010): 34A08, 34A40, 26D10, 33E12.

Received : 18.05.2015 Accepted : 01.04.2016 Doi : 10.15672/HJMS.20164517216

\section{Introduction}

In this paper, we present a Lyapunov's inequality for the following boundary value problem:

$$
\left\{\begin{array}{l}
\left({ }_{a} D^{\alpha} u\right)(t)+q(t) u(t)=0, \quad a<t<b, \quad 2<\alpha \leq 3, \\
u(a)=u^{\prime}(a)=u^{\prime}(b)=0,
\end{array}\right.
$$

where $a$ and $b$ are consecutive zeros of the solution $u$. As $u=0$ is a trivial solution, only non-negative solutions are taken in consideration.

We prove that problem (1.1) has a non-trivial solution for $\alpha \in(2,3]$ provided that the real and continuous function $q$ satisfies

$$
\int_{a}^{b}|q(t)| d t>\frac{\Gamma(\alpha)}{(b-a)^{(\alpha-1)}}\left(\frac{\alpha-1}{\alpha-2}\right)^{\alpha-2} .
$$

${ }^{*}$ College of Sciences, Mathematics department, King Saud University, Riyadh, Saudi-Arabia, Email : maysaa@ksu.edu.sa

${ }^{\dagger}$ Mathematics Department, College of Computers and Information Systems, Al Yamamah University, Riyadh, Saudi Arabia,

Email : radhkla@hotmail.com

$\ddagger_{\text {Corresponding Author. }}$ 
Before we prove this result, let us dwell upon some references.

For the problem

$$
\left\{\begin{array}{l}
u^{\prime \prime}(t)+q(t) u(t)=0, \quad a<t<b \\
u(a)=u(b)=0,
\end{array}\right.
$$

where $a$ and $b$ are consecutive zeros of $u$ and the function $q \in C([a, b] ; \mathbb{R})$. Lyapunov [7] proved a necessary condition of existence of non-trivial solutions is that

$$
\int_{a}^{b}|q(t)| d t>\frac{4}{b-a} .
$$

After this result, similar type inequalities have been obtained for other kind of differential equations and boundary conditions see [3], [8].

Concerning differential equation with fractional derivative's in [2], Ferreira derived Lyapunov's inequality for the problem

$$
\left\{\begin{array}{l}
\left({ }_{a} D^{\alpha} u\right)(t)+q(t) u(t)=0, \quad a<t<b, 1<\alpha \leq 2, \\
u(a)=u(b)=0,
\end{array}\right.
$$

where $q \in C([a, b], \mathbb{R}), a$ and $b$ are consecutive zeros of $u$, and ${ }_{a} D^{\alpha}$ is the RiemannLiouville fractional derivative of order $\alpha>0$ defined for an absolute continuous function on $[a, b]$ by

$$
\left({ }_{a} D^{\alpha} f\right)(t)=\frac{1}{\Gamma(1-\alpha)} \frac{d^{n}}{d t^{n}} \int_{a}^{t}(t-s)^{\alpha} f(s) d s
$$

where $n \in \mathbb{N}, n<\alpha \leq n+1$ (For more details of fractional derivatives see [6]). His inequality reads

$$
\int_{a}^{b}|q(t)| d t>\Gamma(\alpha)\left(\frac{4}{b-a}\right)^{\alpha-1}=\Gamma(\alpha)\left(\frac{2^{2(\alpha-1)}}{(b-a)^{(\alpha-1)}}\right),
$$

which in the particular case $\alpha=2$ corresponds to Lyapunov's classical inequality (1).

Then, Ferreira [3] and Jleli and Samet [5] dealt with fractional differential boundary value problems with Caputo's derivative which is defined for a function $f \in A C^{n}[a, t]$ by

$$
\left({ }_{a}^{C} D^{\alpha} f\right)(t)=\frac{1}{\Gamma(1-\alpha)} \int_{a}^{t}(t-s)^{\alpha} f^{(n)}(s) d s .
$$

For the boundary value problem

$$
\left\{\begin{array}{l}
\left({ }_{a}^{C} D^{\alpha} u\right)(t)+q(t) u(t)=0, \quad a<t<b, 1<\alpha \leq 2 \\
u(a)=u(b)=0
\end{array}\right.
$$

where $q \in C([a, b] ; \mathbb{R})$ and $a$ and $b$ are consecutive zeros of $u$, Ferreira [2] proved that if (1.6) has a nontrivial solution, then the following necessary condition is satisfied

$$
\int_{a}^{b}|q(t)| d t>\frac{\Gamma(\alpha) \alpha^{\alpha}}{[(\alpha-1)(b-a)]^{\alpha-1}} .
$$

In [5], Jleli and Samet considered the equation (1.6) subject to either

$$
u^{\prime}(a)=0, u(b)=0
$$

or

$$
u(a)=0, u^{\prime}(b)=0 .
$$

They showed that the associated non trivial solution exists if

$$
\int_{a}^{b}(b-s)^{\alpha-2}|q(t)| d t \geq \Gamma(\alpha)
$$


is satisfied.

However, in the case of (1.9), the corresponding nontrivial solution exists if:

$$
\int_{a}^{b}(b-s)^{\alpha-2}|q(t)| d t \geq \frac{\Gamma(\alpha)}{\max \{\alpha-1,2-\alpha\}(b-a)} .
$$

It was shown in [4] that a non trivial solution corresponding to equation (1.6) where $q \in C([a, b] ; \mathbb{R}), a$ and $b$ are consecutive zeros of $u$, subject to the boundary conditions

$$
u(a)-u^{\prime}(a)=u(b)+u^{\prime}(b)=0,
$$

exists if the following necessary condition

$$
\int_{a}^{b}(b-s)^{\alpha-2}(b-s+\alpha-1)|q(s)| d s \geq \frac{(b-a+2) \Gamma(\alpha)}{\max \left\{b-a+1, \frac{2-\alpha}{\alpha-1}(b-a)-1\right\}}
$$

is satisfied.

\section{Main results}

2.1. A Lyapunov-type inequality for problem (1.1). The strategy in getting Lyapunovtype inequality for (1.1) is to re-write the considered problem in its equivalent integral form.

As in [2], the solution can be written in the integral form

$$
u(t)=\int_{a}^{t} G(t, s) q(s) u(s) d s+\int_{t}^{b} G(t, s) q(s) u(s) d s
$$

where the Green function $G(x, t)$ is defined by

$$
\begin{gathered}
\Gamma(\alpha) G(t, s)= \begin{cases}\frac{(t-a)^{\alpha-1}}{(b-a)^{\alpha-2}}(b-s)^{\alpha-2}-(t-s)^{\alpha-1}, & a \leq s \leq t, \\
\frac{(t-a)^{\alpha-1}}{(b-a)^{\alpha-2}}(b-s)^{\alpha-2}, & t \leq s \leq b .\end{cases} \\
= \begin{cases}g_{1}(t, s), & a \leq s \leq t \leq b, \\
g_{2}(t, s), & a \leq t \leq s \leq b,\end{cases}
\end{gathered}
$$

which in the particular case $a=0, b=1$ corresponds to that of M. El-Shahed [1].

2.1. Theorem. The Green function $G$ satisfies:

(1)

$$
G(t, s) \geq 0 \text { for all } a \leq t, s \leq b .
$$

(2) $\max _{t \in[a, b]} G(t, s)=G(b, s), \quad s \in[a, b]$,

(3) $G(b, s)$ has a unique maximum given by:

$$
\max _{s \in[a, b]} G(b, s)=\frac{1}{\Gamma(\alpha)}(b-a)^{(\alpha-1)}\left(\frac{\alpha-2}{\alpha-1}\right)^{\alpha-2} .
$$

Proof. For the proof of Theorem 2.1, we start with the function $g_{1}(t, s)$. The function $g_{1}$ is non-decreasing. Indeed, to show this fact, we need to make the following observation of Ferreira in [2]:

$$
\left(a+\frac{(s-a)(b-a)}{t-a}\right) \geq s \text { is equivalent to } s \geq a
$$


this allows us to write

$$
\begin{aligned}
(t-s)^{\alpha-1}=(t-a+a-s)^{\alpha-1} & =\left[(t-a)\left(1+\frac{a-s}{t-a}\right)\right]^{\alpha-1} \\
& =\left[(b-a)\left(1+\frac{a-s}{t-a}\right)\right]^{\alpha-1} \frac{(t-a)^{\alpha-1}}{(b-a)^{\alpha-1}} \\
& =\left[b-\left(a+\frac{(s-a)(b-a)}{t-a}\right)\right]^{\alpha-1} \frac{(t-a)^{\alpha-1}}{(b-a)^{\alpha-1}},
\end{aligned}
$$

which is used to show that $g_{1}$ is positive and non-decreasing.

Indeed,

For $a \leq s \leq t \leq b$,

$$
\begin{aligned}
g_{1}(t, s) & :=\frac{(t-a)^{\alpha-1}}{(b-a)^{\alpha-2}}(b-s)^{\alpha-2}-(t-s)^{\alpha-1} \\
& =\frac{(t-a)^{\alpha-1}}{(b-a)^{\alpha-2}}(b-s)^{\alpha-2}-\left[b-\left(a+\frac{(s-a)(b-a)}{t-a}\right)\right]^{\alpha-1} \frac{(t-a)^{\alpha-1}}{(b-a)^{\alpha-2}} \\
& \geq \frac{(t-a)^{\alpha-1}}{(b-a)^{\alpha-2}}(b-s)^{\alpha-2}-(b-s)^{\alpha-2} \frac{(t-a)^{\alpha-1}}{(b-a)^{\alpha-2}} \\
& \geq 0 .
\end{aligned}
$$

On the other hand

$$
\begin{aligned}
\frac{\partial g_{1}}{\partial t}(t, s)= & (\alpha-1) \frac{(t-a)^{\alpha-2}}{(b-a)^{\alpha-2}}(b-s)^{\alpha-2}-(\alpha-1)(t-s)^{\alpha-2} \\
= & (\alpha-1) \frac{(t-a)^{\alpha-2}}{(b-a)^{\alpha-2}}(b-s)^{\alpha-2}-(\alpha-1)\left[b-\left(a+\frac{(s-a)(b-a)}{t-a}\right)\right]^{\alpha-2} \\
& \frac{(t-a)^{\alpha-2}}{(b-a)^{\alpha-2}} \\
= & (\alpha-1)(t-a)^{\alpha-2}\left(\frac{(b-s)^{\alpha-2}}{(b-a)^{\alpha-2}}-\left[b-\left(a+\frac{(s-a)(b-a)}{t-a}\right)\right]^{\alpha-2} \frac{1}{(b-a)^{\alpha-2}}\right) \\
\geq & (\alpha-1)(t-a)^{\alpha-2}\left(\frac{(b-s)^{\alpha-2}}{(b-a)^{\alpha-2}}-(b-s)^{\alpha-2} \frac{1}{(b-a)^{\alpha-2}}\right) \\
\geq & 0 .
\end{aligned}
$$

Consequently,

$$
\max _{t, s \in[a, b]} g_{1}(t, s)=\max _{s \in[a, b]} g_{1}(b, s) .
$$

In view of $(2.1)-(2.2), g_{1}(b, s)$ is defined by: $g_{1}(b, s)=(b-s)^{\alpha-2}(s-a)$. Its derivative with respect to $s$ takes the form

$$
\begin{gathered}
\frac{\partial g_{1}}{\partial s}=(b-s)^{\alpha-3}[s(1-\alpha)+a(\alpha-2)+b] . \\
\frac{\partial g_{1}}{\partial s}=0 \Leftrightarrow s=s_{*}=\frac{a(\alpha-2)+b}{\alpha-1} .
\end{gathered}
$$

Hence

$$
\max _{s \in[a, b]} g_{1}\left(b, s_{*}\right)=(b-a)^{\alpha-1}\left(\frac{\alpha-2}{\alpha-1}\right) .
$$

The function $g_{2}$ is clearly positive and non decreasing in $t$, so

$$
\max _{t, s \in[a, b]} g_{2}(t, s)=\max _{s \in[a, b]} g_{2}(b, s)=g_{2}(s, s)=\frac{(s-a)^{\alpha}}{(b-a)^{\alpha-2}}=: F(s) .
$$


The function $F$ is increasing for

$$
s \leq s^{*}=\frac{(\alpha-2) a+(\alpha-1) b}{2 \alpha-3} ;
$$

and is decreasing for

$$
s \geq s^{*}=\frac{(\alpha-2) a+(\alpha-1) b}{2 \alpha-3}
$$

So

$$
\max F(s)=\max g_{2}(s, s)=g_{2}\left(s^{*}, s^{*}\right),
$$

where

$$
g_{2}\left(s^{*}, s^{*}\right)=(b-a)^{\alpha-1}\left(\frac{\alpha-2}{\alpha-1}\right)^{\alpha-2} .
$$

Now we need to compare $g_{1}\left(b, s_{*}\right)$ and $g_{2}\left(s^{*}, s^{*}\right)$.

Since $2 \leq \alpha \leq 3$ then $(2 \alpha-3)^{\alpha-3} \geq(\alpha-1)^{2 \alpha-3}$ and therefore

$$
(b-a)^{\alpha-1}\left(\frac{\alpha-2}{\alpha-1}\right)^{\alpha-2} \geq(b-a)^{\alpha-1} \frac{(\alpha-1)^{\alpha-1}(\alpha-2)^{\alpha-2}}{(2 \alpha-3)^{2 \alpha-3}} .
$$

Consequently

$$
\max _{s \in[a, b]} G(b, s)=\frac{1}{\Gamma(\alpha)}(b-a)^{(\alpha-1)}\left(\frac{\alpha-2}{\alpha-1}\right)^{\alpha-1} .
$$

We are now ready to prove the Lyapunov's type-inequality for problem (1.1).

2.2. Theorem. Let $u$ be a solution satisfying the following boundary value problem

$$
\left\{\begin{array}{l}
\left({ }_{a} D^{\alpha} u\right)(t)+q(t) u(t)=0, \quad a<t<b, 2<\alpha \leq 3 \\
u(a)=u^{\prime}(a)=u^{\prime}(b)=0
\end{array}\right.
$$

where $a$ and $b$ two consecutive zeros of $u$. Then (2.3) has a non-trivial solution provided that the real and continuous function $q$ satisfies the condition

$$
\int_{a}^{b}|q(t)| d t>\frac{\Gamma(\alpha)}{(b-a)^{\alpha-1}}\left(\frac{\alpha-1}{\alpha-2}\right)^{\alpha-1}
$$

Proof. For the proof of Theorem 2.2, we equip the Banach space $C[a, b]$ with the Chebychev norm $\|u\|=\max _{t \in[a, b]}|u(t)|$.

As

we have

$$
u(t):=\int_{a}^{b} G(t, s) q(s) u(s) d s,
$$

$$
\|u\| \leq \int_{a}^{b} \max _{t, s \in[a, b]}|G(t, s)||q(s)| d s\|u\| .
$$

Then since $u$ is a non trivial solution, in view of Theorem 2.1, we get

$$
1 \leq \int_{a}^{b} \frac{1}{\Gamma(\alpha)}(b-a)^{(\alpha-1)}\left(\frac{\alpha-2}{\alpha-1}\right)^{\alpha-1}|q(s)| d s .
$$

Using the properties of $G$, the inequality (2.4) is obtained.

Acknowledgement. This research project was supported by a grant from the Research Center of the Female Scientific and Medical Colleges, Deanship of Scientific Research, King Saud University. 


\section{References}

[1] El-Shahed, M., Positive solutions for boundary value problem of nonlinear fractional differential equation, Abstr. Appl. Anal., Article ID 10368, 8 pp., 2007.

[2] Ferreira, R. A. C., A Lyapunov-type inequality for a fractional boundary value problem, Fract. Calc. Appl. Anal. 16 (4), 978-984, 2013.

[3] Ferreira, R. A. C., On a Lyapunov-type inequality and the zeros of a certain Mittag-Leffler function, J. Math. Anal. Appl. 412, no. 2, 1058-1063, 2014.

[4] Jleli, M., Ragoub, L., Samet, B., A Lyapunov-type inequality for a fractional differential equation under a Robin boundary condition, J. Funct. Spaces, Volume 2015, Article ID 468536, 5 pp., 2015.

[5] Jleli, M., Samet, B., Lyapunov-type inequality for a fractional differential equation with a mixed boundary condition, Math. Ineq. Appl., vol. 18, no. 2, pp. 443-451, 2015.

[6] Kilbas, A. A., Srivastava, H. M., Trujillo, J. J., Theory and applications of fractional differential equations, vol. 204 of North-Holland Mathematics Studies, Elsevier, Amsterdam, $\mathrm{xvi}+523$ pp., 2006.

[7] Lyapunov, A. M., Problème général de la stabilité du mouvement, Ann. Fac. Sci. Univ. Toulouse. 2, 203-407, 1907.

[8] Pachpatte, B. G., Mathematical Inequalities, North Holland Mathematical Library 67, Elsevier, xii +591 pp, 2005. 